\title{
Signal Remodulation of OFDM-QAM for Long Reach Carrier Distributed Passive Optical Networks
}

\author{
C. W. Chow, Member, IEEE, C. H. Yeh, C. H. Wang, Student Member, IEEE, F. Y. Shih, Student Member, IEEE, \\ and S. Chi
}

\begin{abstract}
The passive optical network (PON) using orthogonal frequency-division multiplexing (OFDM) is a subject of many research works recently. The OFDM signal is especially good for long reach (LR)-PON due to its high tolerance to chromatic dispersion and high spectral efficiency. We study, for the first time, the possibility of using OFDM for signal remodulation in LR-PONs. Three different colorless optical networking unit (ONU) architectures, electroabsorption modulator-based, reflective semiconductor optical amplifier-based, and injection-locked Fabry-Pérot laser diode-based ONUs, are tested and compared. Error-free operations are achieved in $100-\mathbf{k m}$ fiber transmission without dispersion compensation.
\end{abstract}

Index Terms-Orthogonal frequency-division multiplexing (OFDM), passive optical networks (PONs), signal remodulation.

\section{INTRODUCTION}

$\mathbf{T}$ O economically sustain the growth of broadband services for end users, it would be required that the price of bandwidth reduces with volume at a rate which has not been achieved by any technology. Since the underlying optical components [e.g., lasers and optical receivers (Rxs)] cannot price decline at the required rate, the only answer to reduce the cost is to reduce the amount of equipment in the network. The long reach (LR) $(>100 \mathrm{~km})$ passive optical network (PON) using dense wavelength-division-multiplexing (DWDM) technology is considered as a promising candidate for the future [1]-[3]. These LR-PONs can reduce the number of network elements and interconnection interfaces, and are predicted to significantly reduce the capital and operational cost. Although LR-PONs are as yet a research concept, the potential benefits they offer are such that they are a very worthy research topic [1]-[3].

Manuscript received January 05, 2009; revised February 13, 2009. First published March 16, 2009; current version published May 15, 2009. This work was supported by the National Science Council, Taiwan, R.O.C., under Contract NSC 96-2218-E-009-025-MY2, 97-2221-E-009-038-MY3.

C. W. Chow, C. H. Wang, and F. Y. Shih are with the Department of Photonics, National Chiao Tung University, Hsinchu 30010, Taiwan (e-mail: cwchow@faculty.nctu.edu.tw; shoutclose@gmail.com; yuan0253@hotmail.com).

C. H. Yeh is with the Information and Communications Research Laboratories, Industrial Technology Research Institute, 31040, Taiwan (e-mail: depew@itri.org.tw).

S. Chi is with the Department of Photonics, National Chiao Tung University, Hsinchu 30010, Taiwan. He is also with the Department of Electro-Optical Engineering, Yuan Ze University, Chungli 32003, Taiwan (e-mail: schi@mail. nctu.edu.tw).

Color versions of one or more of the figures in this letter are available online at http://ieeexplore.ieee.org.

Digital Object Identifier 10.1109/LPT.2009.2017205
Since there are different distances between the head-end office and the customer optical networking units (ONUs) in these LR-PONs, it is very difficult to fully compensate the chromatic dispersion. Moreover, due to the high split-ratio $(>100)$ of the LR-PON, high data rate is required to support the high split-ratio. Hence chromatic dispersion is one of the severe issues. Recently, we proposed using an orthogonal frequency-division-multiplexed (OFDM) signal for the LR, high split-ratio access network [4]. The inherent advantage of OFDM frequency diversity transmission allows effectively mitigation of chromatic dispersion. In addition, due to the highly spectral efficiency, low-bandwidth optical components can still be used. We have demonstrated that $4 \mathrm{~Gb} / \mathrm{s}$ of 16-quadrature amplitude modulation (QAM)-OFDM signal can be carried in the LR-PON using standard gigabit passive optical network (bandwidth of $\sim 1 \mathrm{GHz}$ ) optical components. A split-ratio of 256 was achieved. We also analyzed that it is possible to use a higher level of QAM, such as 256-QAM [5], to further increase the aggregate data rate approaching $10 \mathrm{~Gb} / \mathrm{s}$.

Here, we extend our analysis to the signal remodulated LR-PON using OFDM signal. Signal remodulation has attracted a lot of research for the past few years as one of the promising candidates for next-generation PON [6]-[8]. An expensive transmitter inside the ONU with a distinct and specific wavelength has hindered the PON deployment. Network architectures with signal remodulation on the downstream channel for the upstream channel can reduce the cost of wavelength referencing, control, and stabilization at the cost-sensitive ONU. A single wavelength is needed for both downstream and upstream signals, since the wavelength is reused. In addition, the same colorless ONU can be used for all the customers.

Here, we study, for the first time, the possibility of using OFDM for signal remodulation in the LR-PON. Three different colorless ONU architectures, electroabsorption modulator (EAM)-based, reflective semiconductor optical amplifier (RSOA)-based, and injection-locked Fabry-Pérot laser diode (FP-LD)-based ONUs, are tested and compared, and error-free operations are achieved.

\section{EXPERIMENTAL SETUP}

Fig. 1 shows the proposed signal remodulation LR-PON. A baseband digital signal processing was used to produce the 16-QAM OFDM signal. An arbitrary waveform generator with 4-GHz sampling rate was then used to convert the digital-to-analog data, which was then applied to the EAM for downstream connection. The 4-Gb/s OFDM signal consisted of 16 subcarriers occupying about $1-\mathrm{GHz}$ bandwidth. Each subcarrier was in 16-QAM format. The 1-G symbol/s OFDM 


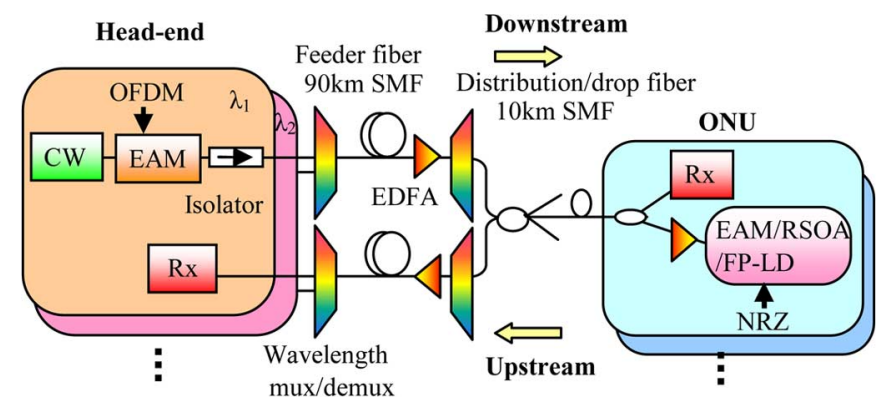

Fig. 1. Experimental setup of signal remodulation DWDM-PON. SMF: singlemode fiber.

signal occupied the radio-frequency spectrum from 62.5 to $1125 \mathrm{MHz}$, with data pattern consisted of 8192 OFDM symbols. The cyclic prefix was $1 / 32$ symbol time. The detail OFDM signal generation was described in [5]. The downstream signal was at wavelength of $1548 \mathrm{~nm}$, with average input power to the feeder fiber of $5 \mathrm{dBm}$. The signal was then propagating through 90 and $10 \mathrm{~km}$ of feeder and distribution/drop fibers, respectively, via a pair of arrayed waveguide grating (AWG) [Gaussian shaped, 100-GHz channel spacing] and an erbium-doped fiber amplifier (EDFA), which is used to compensate the loss during transmission. At the ONU, $10 \%$ of downstream power was tapped out and detected by an optically preamplified $\mathrm{Rx}$, which consisted of a low-noise EDFA, optical bandpass fiber to remove the out-of-band amplified spontaneous emission, and a PIN photodiode. An analog-to-digital converter, which is a real-time $10-\mathrm{GHz}$ sampling oscilloscope, converted the downstream signal detected by the optically preamplified $\mathrm{Rx}$ to digital signal for demodulation. The demodulation was performed using computer software, and it consisted of a synchronizer to extract the carrier phase and aligned the OFDM symbol boundaries, a fast Fourier transform to translate signal from time to frequency domain, and a QAM decoder to analyze the symbol on each subcarrier to make the final decision. The bit-error rate (BER) was calculated from the measured error vector magnitude [9], [10].

The remaining downstream signal was then launched into the colorless modulator at the ONU. The optical amplifier was placed in front of the colorless modulator in each ONU to compensate the loss and to provide enough optical power for the colorless modulator. In this research, we analyzed three different kinds of colorless modulators: EAM, RSOA, and FP-LD. In each case, a 2.5-Gb/s nonreturn-to-zero (NRZ) data, pseudorandom binary sequence pattern length of $2^{31}-1$, was used to directly modulate the colorless modulator. Then the upstream signal was launched back to the head-end Rx for BER measurements through the second feeder fiber.

\section{RESULTS AND DISCUSSION}

We first study the optimum extinction ratio (ER) of the downstream OFDM-QAM signal for both downstream and upstream links. The ER is defined as $10 \log _{10}\left(P_{1} / P_{0}\right)$, where $P_{1}$ and $P_{0}$ are the maximum and minimum power of the OFDM signal, respectively. The input power to each ONU was $\sim-3 \mathrm{dBm}$. As shown in Fig. 2, by decreasing the ER of the OFDM signal,

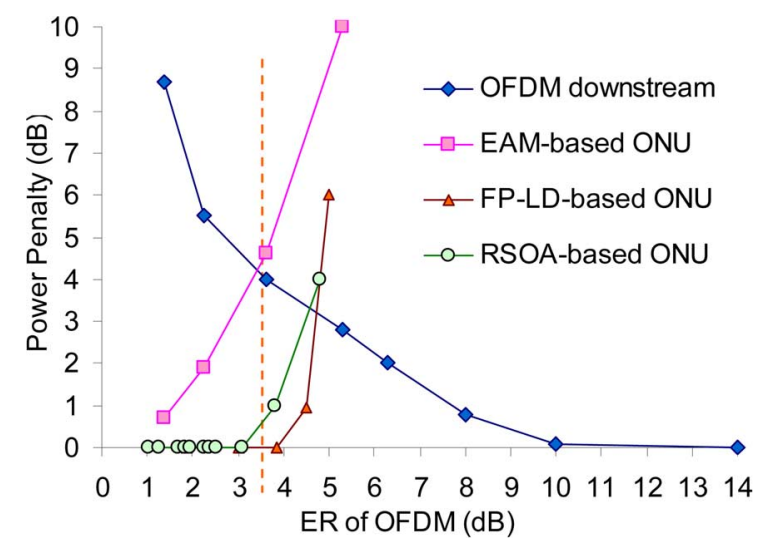

Fig. 2. Power penalties of downstream OFDM and upstream NRZ signals generated by different kinds of ONUs against different ER of the downstream OFDM signal.

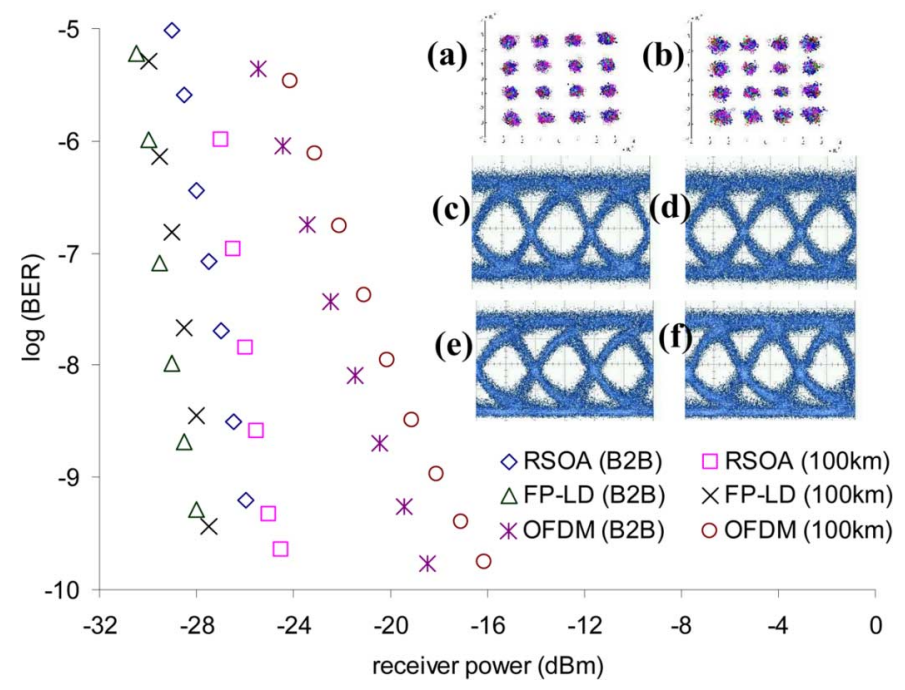

Fig. 3. BER measurements of different signals. Insets: constellation diagrams of OFDM signal at $\mathrm{ER}=3.7 \mathrm{~dB}$ at (a) B2B, (b) $100 \mathrm{~km}$, output remodulated NRZ eye diagrams of FP-LD at (c) B2B, (d) $100 \mathrm{~km}$; and RSOA at (e) B2B, (f) $100 \mathrm{~km}$, when downstream OFDM at $\mathrm{ER}=3.7 \mathrm{~dB}$.

higher residual background light can be provided for the upstream remodulation. When an EAM-based ONU is used, the intercepting point is at the position where the ER of OFDM is equal to $\sim 3.7 \mathrm{~dB}$, and it means $4 \mathrm{~dB}$ of power penalty will be introduced to both the upstream and downstream signals. We can see that RSOA-based and FP-LD-based ONUs performed better. By using the gain-saturation property of the RSOA or the injection locking property of the FP-LD, the downstream OFDM signal can be significantly suppressed, and these can increase the intercepting point to $\sim 4.7 \mathrm{~dB}$. This shows a power penalty improvement of $1 \mathrm{~dB}$ in the OFDM signal.

Then, we tested the three kinds of colorless ONUs for the signal remodulation LR-PON. Since each modulator has its optimum condition, in the comparison, we selected the $\mathrm{ER}=3.7 \mathrm{~dB}$ for the downstream OFDM signal. Fig. 3 shows the BER measurements of each signal at back-to-back (B2B) and after the 100-km LR transmission. Insets show the constellation diagrams of OFDM signal at $\mathrm{ER}=3.7 \mathrm{~dB}$, output remodulated NRZ eye diagrams of FP-LD, and RSOA when 

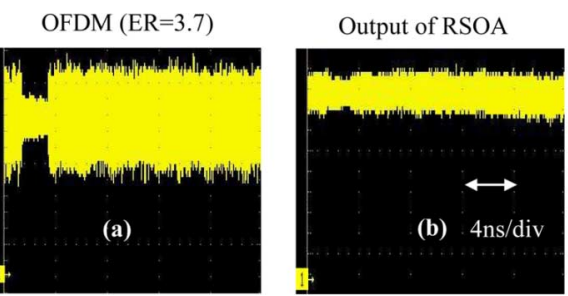

Output of FP-LD

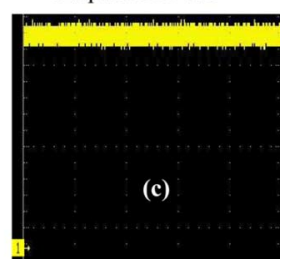

Fig. 4. Time traces from the real-time oscilloscope of (a) the OFDM, output of (b) the RSOA and (c) FP-LD when the ER of the input OFDM signal is $3.7 \mathrm{~dB}$.
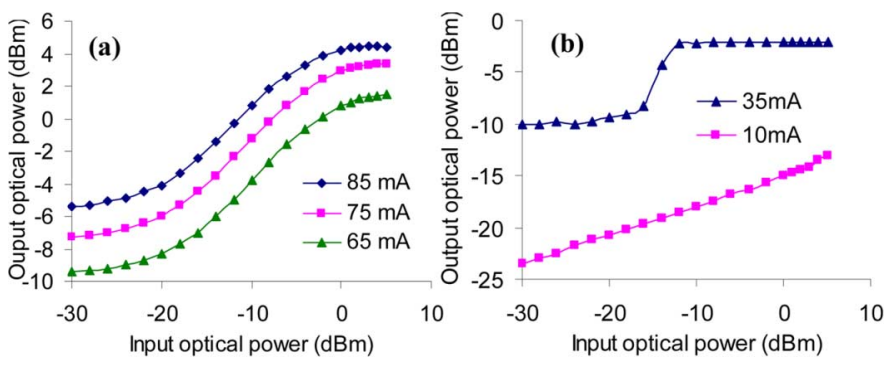

Fig. 5. Output optical powers against different input optical powers for the (a) RSOA- and (b) FP-LD-based ONU under different dc biases.

downstream OFDM at ER $=3.7 \mathrm{~dB}$. We can observe clear open eyes after 100-km single-mode fiber transmission without dispersion compensation in each case. Error-free BER operation can be achieved for the proposed remodulation LR system. In this proof-of-concept experiment, only a single ONU was studied.

We further compared the performance of the RSOA-based and FP-LD-based colorless ONU for the remodulation system. In Fig. 2, we observe that the threshold property of the injection locking can provide a better upstream NRZ signal (less power penalty in the BER analysis) for the same downstream OFDM signal. For better understanding, we then observed using time traces. Fig. 4 show the time traces from the real-time oscilloscope of (a) the downstream OFDM $(\mathrm{ER}=3.7 \mathrm{~dB})$, output of (b) the RSOA and (c) FP-LD when the ER of the input OFDM signal is $3.7 \mathrm{~dB}$. The electrical data applied to the RSOA or FP-LD was switched OFF and only dc bias was used. We observe clearly that the injection locking can suppress the downstream OFDM signal much higher than in the case of RSOA. After this, we studied the nonlinear transmission characteristics of the RSOA- and FP-LD-based colorless ONUs. Different continuous-wave optical powers were launched into them at different biases, and we measured the output optical powers using a power meter, as shown in Fig. 5(a) and (b), respectively, for the RSOA and FP-LD. We can observe that the nonlinear transfer function of the RSOA-based ONU is less steep than that of the FP-LD-based ONU; and the shape of the RSOA curves are similar under different dc biases. For the injection-locked FP-LDbased ONU, when the FP-LD is biased below the threshold current $(10 \mathrm{~mA})$, the response is quite linear. When the biased current is much above threshold $(35 \mathrm{~mA})$, the nonlinear transfer function is much steeper than that of the RSOA-based ONU. Hence, the downstream OFDM signal can be highly suppressed by the injection-locking of the FP-LD-based ONU to produce a better upstream NRZ signal. However, it is also worth mentioning that since injection-locking depends on input polarization, and the random birefringence of buried optical fiber networks typically causes slow fluctuations in the polarization angles of the propagating signals [11], a slow dynamic polarization control may be used to compensate the change in polarization state [12].

\section{CONCLUSION}

PON using OFDM is a subject of many research works recently. The OFDM signal is a promising candidate for LR-PON due to its high tolerance to chromatic dispersion. Here, we studied the possibility of using OFDM for signal remodulation in the LR-PON. Three different colorless ONU architectures, EAM-based, RSOA-based, and FP-LD-based, were tested and compared, showing that despite the polarization controlling issue, FP-LD can provide a greater downstream OFDM suppression and produce better upstream data.

\section{REFERENCES}

[1] D. P. Shea, A. D. Ellis, D. B. Payne, R. P. Davey, and J. E. Mitchell, "10 Gbit/s PON with $100 \mathrm{~km}$ reach and x1024 split," in Proc. ECOC, Rimini, Italy, 2003, Paper We.P.147.

[2] P. D. Townsend, G. Talli, C. W. Chow, E. M. MacHale, C. Antony, R. Davey, T. De Ridder, X. Z. Qiu, P. Ossieur, H. G. Krimmel, D. W. Smith, I. Lealman, A. Poustie, S. Randel, and H. Rohde, "Long reach passive optical networks," in IEEE LEOS Annual Meeting, Florida, 2007.

[3] G. Talli, C. W. Chow, E. K. MacHale, and P. D. Townsend, "Long reach hybrid DWDM-TDM PON with high split ratio employing centralized light source," J. Opt. Netw., vol. 6, pp. 765-776, 2007.

[4] C. W. Chow, C. H. Yeh, C. H. Wang, F. Y. Shih, Y. M. Lin, and S. Chi, "Demonstration of high spectral efficient OFDM-QAM long reach passive optical networks," in Proc. ECOC, Belgium, 2008, Paper Th.2.F.5

[5] C. W. Chow, C. H. Yeh, C. H. Wang, F. Y. Shih, C. L. Pan, and S. Chi, "WDM extended reach passive optical networks using OFDM-QAM," Opt. Express, vol. 16, pp. 12096-12101, 2008.

[6] L. Y. Chan, C. K. Chan, D. T. K. Tong, F. Tong, and L. K. Chen, "Upstream traffic transmitter using injection-locked Fabry-Perot laser diode as modulator for WDM access networks," Electron. Lett., vol. 38, pp. 43-45, 2002.

[7] W. Hung, C. K. Chan, L. K. Chen, and F. Tong, "An optical network unit for WDM access networks with downstream DPSK and upstream remodulated OOK data using injection-locked FP laser," IEEE Photon. Technol. Lett., vol. 15, no. 10, pp. 1476-1478, Oct. 2003.

[8] C. W. Chow, "Wavelength remodulation using DPSK down-and-upstream with high extinction ratio for 10-Gb/s DWDM-passive optical networks," IEEE Photon. Technol. Lett., vol. 20, no. 1, pp. 12-14, Jan. $1,2008$.

[9] Y. M. Lin, "Demonstration and design of high spectral efficiency 4 Gb/s OFDM system in passive optical networks," in Proc. OFC, Anaheim, CA, 2007, Paper OThD7.

[10] V. J. Urick, J. X. Qiu, and F. Bucholtz, "Wide-band QAM-over-fiber using phase modulation and interferometric demodulation," IEEE Photon. Technol. Lett., vol. 16, no. 10, pp. 2374-2376, Oct. 2004.

[11] G. Nicholson and D. J. Temple, "Polarization fluctuation measurements on installed single-mode optical fiber cables," J. Lightw. Technol., vol. 7, no. 8, pp. 1197-1200, Aug. 1989.

[12] F. Heismann and M. S. Whalen, "Fast automatic polarization control system,” IEEE Photon. Technol. Lett., vol. 4, no. 5, pp. 503-505, May 1992. 